Materiales de Construcción

Vol. 60, 299, 53-67

julio-septiembre 2010

ISSN: 0465-2746

eISSN: $1988-3226$

doi: $10.3989 / \mathrm{mc} .2010 .49708$

\title{
Cortante-fricción de los hormigones reciclados
}

\section{Shear friction capacity of recycled concretes}

\author{
B. González Fonteboa ${ }^{(*)}$, F. Martínez ${ }^{(*)}$, D. Carro(*), J. Eiras ${ }^{(*)}$
}

Recepción/Received: 15-XII-2008

Aceptación/Accepted: 1-VI-2009

Publicado online/Online publishing: 11-VIII-2010

\section{RESUMEN}

En esta investigación se estudió el comportamiento de los hormigones reciclados frente al fenómeno de transmisión de cortante. Para ello se diseñó un hormigón convencional de control y un hormigón con el $50 \%$ del árido grueso reciclado. Adicionalmente, para determinar cómo este comportamiento se ve modificado con la incorporación de una adición puzolánica (humo de sílice), se procedió al diseño de un hormigón convencional y su correspondiente reciclado con un $8 \%$ de humo de sílice.

Los resultados indicaron una disminución de la capacidad frente a este fenómeno en los hormigones reciclados, más acusada en ausencia de armadura pasante. La adición de humo de sílice mejora el comportamiento de este material.

Los resultados experimentales obtenidos se compararon con formulaciones teóricas de diversos autores, concluyéndose que éstas son, en todos los casos, conservadoras, aunque reducen el margen de seguridad en los hormigones reciclados.

Palabras clave: humo de sílice, hormigón reciclado, árido reciclado. cortante-fricción, deformación.

\section{SUMMARY}

The aim of this research was to determine the behavior of recycled concrete in response to the phenomenon of shear transfer. To perform it, a conventional control concrete and a concrete with $50 \%$ recycled coarse aggregate were designed. An additional goal was to shed light on how this behavior is modified with a pozzolanic addition, silica fume. Therefore, two types of concrete were designed, a conventional and a recycled concrete, both made with $8 \%$ of silica fume.

In conclusion, a reduction of shear friction capacity was observed in recycled concretes, considerably higher in the case of the specimen without reinforcement. The addition of silica fume improved the behavior of recycled concretes.

The results obtained were compared with the formulations of the different authors. In all cases, these were found to be conservative. However, the safety margins offered by recycled concretes are lower than those obtained with conventional concretes.

Keywords: silica fume, recycled concrete, recycled aggregate, shear friction, strain.

(*) Universidad de A Coruña (A Coruña, España). 


\section{INTRODUCCIÓN}

El término cortante-fricción se utiliza para describir el mecanismo de transmisión de cortante entre caras de un plano de hormigón sometido de forma simultánea a solicitaciones de cortante y de compresión. Los factores que modifican la capacidad del hormigón frente a este fenómeno se describen a continuación:

- Engranamiento de áridos y fricción: resultado de la rugosidad entre caras de fisura. Esta contribución puede ser local, cuando afecta a una fisura de longitud inferior al tamaño de los áridos o global, para longitudes superiores.

- Solicitación normal de compresión entre caras de fisura: aumenta la contribución del engranamiento de áridos y la fricción.

- Armadura pasante entre caras del plano de ensayo:

- Deformación de armadura: moviliza esfuerzos de compresión entre caras del plano de ensayo que incrementan la contribución de la solicitación externa.

- Efecto dovela: el desplazamiento entre caras del plano de ensayo supone la aparición de esfuerzos de flexión y cortante frente a los que actúa la armadura pasante.

- Resistencia del hormigón: la capacidad de transmisión de cortante entre caras del plano de ensayo se pierde cuando el contacto entre éstas se anula. Esto ocurre cuando se supera la resistencia de la pasta o de los áridos y está directamente relacionado con la resistencia del hormigón.

Desde 1960 se han desarrollado numerosas investigaciones sobre el comportamiento de los hormigones convencionales frente a cortante-fricción. Gran parte de éstas pretendían determinar los parámetros que influyen en el fenómeno realizando ensayos "push-off". En este tipo de ensayo se introduce un cortante puro y una solicitación normal de compresión en el plano de rotura, que puede ser real (una probeta hormigonada sobre otra) o ficticia (la probeta se hormigona monolíticamente) (1-3).

\section{OBJETIVOS}

En hormigón convencional las fisuras atraviesan la matriz de la pasta, propagándose alrededor de las partículas, más resistentes, del árido (roturas intergranulares), que embebidas en la matriz débil de la pasta, permiten la transmisión de esfuerzos a través de numerosos puntos de contacto. En otros hormigones, como los hormigones de alta resistencia o los hormigones ligeros, las fisuras atraviesan, también, a las partículas del árido (roturas

\section{INTRODUCTION}

Shear friction is the term used to describe the mechanism of shear transfer along a concrete to concrete interface subjected to simultaneous shear and normal compression. The factors affecting shear friction capacity can be listed as follows:

- Aggregate interlock and friction: as a result of crack face roughness (local, oscillation taking place at a length smaller than the average aggregate size and global, oscillation taking place on a much longer scale).

- Compression force applied normal to the interface: this increase contribution of aggregate interlock and friction.

- Reinforcement crossing the interface:

- Clamping: elongation of reinforcement across the crack provides compression forces on the interfaces. This contributes to increase external force.

- Dowel action: crack slip causes bending and shear forces in the reinforcement.

- Concrete strength: failure of the interface to transmit shearing forces results from loss of contact, which in turn occurs due to crushing of interlocking aggregates and cement paste. Crushing occurs when constituents reach their capacity, which is directly related to concrete strength.

There has been a great deal of research in this area since 1960 's. Most of the tests carried out to establish shear friction parameters were in the form of push off tests. In these tests, pure shear and a normal force is introduced on a failure plane. This plane can be real (one part of the specimen is cast against the other) or potential (specimen that is cast monolithically) (1-3).

\section{OBJECTIVES}

In conventional concrete the cracks intersect the cement matrix, but propagate around the relatively strong aggregate particles. The strong aggregate particles are pushed into the weaker matrix, and a transmission of forces is generated at numerous contact points. In other concretes (such as high strength concrete or lightweight concretes), the cracks intersect the aggregate particles 
intragranulares), de forma que el número de puntos de contacto se reduce de manera significativa.

Así, en ausencia de armadura pasante, la capacidad frente a cortante-fricción del hormigón de alta resistencia disminuye hasta el $35 \%$ de la capacidad desarrollada por un hormigón convencional. En hormigón de alta resistencia con armadura pasante esta capacidad alcanza el 55$75 \%$ de la obtenida con el convencional (4-5).

El comportamiento de los hormigones reciclados en este aspecto es desconocido. Sin embargo, la presencia de mortero adherido al árido reciclado hace prever un debilitamiento del área de contacto que implicaría una reducción de la capacidad frente a cortante-fricción.

En diciembre de 2008 entró en vigor la nueva EHE, en cuyo anejo 15 se ofrecen las recomendaciones para el empleo de hormigones reciclados. Este anejo establece una sustitución conservadora del $20 \%$ de árido grueso aunque, en algunos casos, se dan pautas para emplear sustituciones mayores. Este trabajo intenta conocer la influencia del porcentaje de sustitución en el comportamiento cortante-fricción, fenómeno en el que el anejo indica que es necesaria una investigación específica y un mayor conocimiento. Se pretende, así, registrar el comportamiento para porcentajes de sustitución del $50 \%$, así como determinar si la adición de una puzolana (humo de sílice) a los hormigones reciclados consigue modificar la respuesta frente a este fenómeno.

\section{ESTADO DEL CONOCIMIENTO}

Se ha realizado una revisión de las ecuaciones que tratan de ajustar el comportamiento de los hormigones frente a cortante-fricción. En la Tabla 1 se detallan las desarrolladas por diversos autores (6-13). Las cuatro primeras proceden de ensayos realizados con hormigones de resistencias a compresión muy similares, de ahí que las expresiones no reflejen el efecto de su variabilidad. Para considerar este efecto se realizaron nuevos ensayos con hormigones de resistencias mayores que cubriesen un mayor rango de variación, surgiendo así las cuatro siguientes ecuaciones de la tabla.

La mayoría de las expresiones han sido desarrolladas para hormigones en los que no se produce rotura del árido por lo que, obviamente, no alcanzan a hormigones no convencionales de forma que, para estos materiales, las ecuaciones podrían resultar inseguras. Sin embargo, en algunos casos (por ejemplo en hormigón ligero), los autores incorporan coeficientes que pretenden tener en cuenta el comportamiento de estos hormigones (es el caso de las ecuaciones [2] [3] [4] y [8]). as well, so the number of contact points is reduced significantly.

It was found that for unreinforced concrete, the shear friction capacity of high strength concrete was around $35 \%$ of that of normal strength concrete. For reinforced concrete this capacity was $55-75 \%$ of that of normal strength concrete (4-5).

In this respect, the behavior of recycled concretes is not known. However, owing to the presence of mortar adhering to the recycled aggregate, a decrease in the contact area would be expected, which would lead to reduced shear friction capacity.

In December 2008 the new EHE has entered in effect. In the section 15 it offers the recommendations for the use of recycled concretes. In it, a conservative substitution of $20 \%$ of coarse aggregate is established, though, in some cases, guidelines to use major substitutions are given. This work tries to know the influence of the percentage of substitution in the shear - friction behavior, phenomenon in which the annex indicates that is necessary a specific investigation and a major knowledge. This research aims to know the behavior of recycled concretes with $50 \%$ of recycled coarse aggregate, as well as to determine if the addition of a mineral admixture (silica fume) to the recycled concretes modifies or not their shear --friction behavior.

\section{REVIEW OF ULTIMATE CAPACITY METHODS}

There has been made a review of the equations that try to fit the shear-friction behavior of the concretes. In the table 1 some of them are detailed. The first four come from tests made with concretes of very similar compressive strength, so the expressions do not reflect the effect of this parameter. To consider it new tests were realized using concretes with compressive strength covering a major range of variation. From these tests all four following equations of the table were developed.

All these equations were developed primarily for specimens where aggregate fracture does not take place. Obviously, this does not include high strength concrete or recycled concrete. In these cases the equations may be unsafe. However, in some instances (eg light concrete), the authors incorporate coefficients to take into account the behavior of these concretes (that is the case of the equations [2] [3] [4] and [8]) 
Tabla 1 / Table 1

Ecuaciones para el fenómeno de cortante-fricción.

Shear friction equations.

\begin{tabular}{|c|c|}
\hline $\begin{array}{l}\text { Birkland \& Birkland (6) } \\
\text { teoría clásica de cortante-fricción / } \\
\text { classical shear friction theory }\end{array}$ & 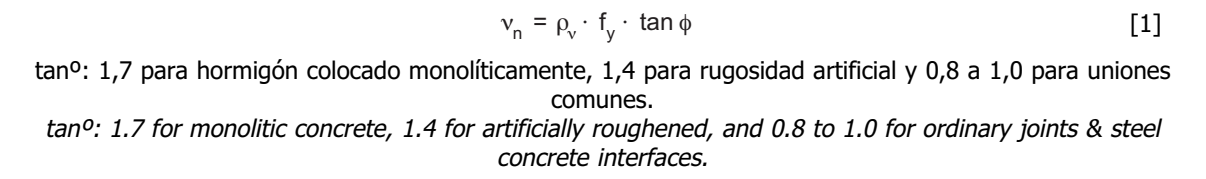 \\
\hline Raths (7) & $\begin{array}{l}\qquad v_{\mathrm{n}}=3.11 \cdot \lambda \cdot \sqrt{\rho_{v} \cdot f_{\mathrm{y}}} \\
\lambda: 1,0 \text { para hormigón convencional, } 0,87 \text { para hormigón con árido fino ligero, y } 0,75 \text { para hormigón ligero. } \\
\lambda: 1 \text { for normal weight concrete, } 0.87 \text { for sand lightweight concrete, and } 0.75 \text { for all lightweight concrete. }\end{array}$ \\
\hline Shaikh (8) & $\begin{array}{c}\qquad v_{\mathrm{n}}=\lambda \cdot \sqrt{6.90 \cdot \mu} \cdot \sqrt{\rho_{v} \cdot f_{\mathrm{y}}} \\
\lambda: 1,0 \text { para hormigón convencional, } 0,87 \text { para hormigón con árido fino ligero, y } 0,75 \text { para hormigón ligero. } \\
\lambda: 1 \text { for normal weight concrete, } 0.87 \text { for sand lightweight concrete, and } 0.75 \text { for all lightweight concrete. } \\
\mu: 1,4 \text { para hormigón colocado monolíticamente, } 1,0 \text { para hormigón colocado sobre hormigón endurecido, } \\
0,4 \text { para uniones poco rugosas y } 0,6 \text { para uniones con armadura pasante. } \\
\mu: 1.4 \text { for monolithic cast concrete, } 1.0 \text { for concrete cast to hardened concrete, } 0.4 \text { for smooth } \\
\text { concrete interfaces and } 0.6 \text { for concrete to steel interfaces. }\end{array}$ \\
\hline $\begin{array}{l}\text { Mattock (9) } \\
\text { teoría cortante-fricción modificada / } \\
\text { shear friction with nonzero tension } \\
\text { cutoff }\end{array}$ & $\begin{array}{c}\qquad v_{\mathrm{n}}=0.8 \cdot \frac{\mathrm{A}_{\mathrm{s}}}{\mathrm{A}_{\mathrm{c}}} \cdot \mathrm{f}_{\mathrm{y}}+\mathrm{K}_{1} \\
\mathrm{~K}_{1}: 2,8 \text { para hormigón convencional, 1,7 para hormigón con árido fino ligero, y 1,5 para hormigón ligero. } \\
K_{1}: 2.8 \mathrm{MPa} \text { for normal weight concrete, } 1.7 \mathrm{MPa} \text { for sand lightweight concrete, and 1.5 MPa for } \\
\text { all lightweight concrete. }\end{array}$ \\
\hline $\begin{array}{l}\text { Walraven, Frénay, } \\
\text { Pruijssers }(10)\end{array}$ & $\begin{array}{c}v_{n}=C_{1} \cdot\left(\rho_{v} \cdot f_{y}\right) C_{2} \quad C_{1}=0.822 \cdot f_{c c}^{0.406} \text { y } \quad C_{2}=0.159 \cdot f_{c c}^{0.303} \\
f_{c c}: \text { resistencia a compresión en probeta cúbica de } 150 \mathrm{~mm} \text { de lado }\left(f_{c k}=0,85 \cdot f_{c c}\right) \\
f_{c c}: \text { compressive strength of the } 150 \mathrm{~mm} \text { cube specimen }\left(f_{c k}=0.85 \cdot f_{c c}\right)\end{array}$ \\
\hline Mattock (11) & $\begin{array}{l}\quad v_{n}=0.467 \cdot f_{c}^{0.545}+0.8\left(\rho_{v} \cdot f_{y}+\sigma_{n}\right) \\
\sigma_{n}: \text { tensión externa normal al plano de la junta. } \\
\sigma_{n}: \text { normal external stress on the plane of the joint. }\end{array}$ \\
\hline Mau, Hsu's (12) & $\frac{v_{n}}{f_{c}}=0.66 \cdot \sqrt{\omega}<03$ con $\omega=\frac{\rho \cdot f_{y}}{f_{c}}$ \\
\hline Loov, Patnaik (13) & $\begin{array}{c}\qquad v_{\mathrm{n}}=\mathrm{k} \cdot \lambda \cdot \sqrt{\left(0.1+\rho_{\mathrm{v}} \cdot \mathrm{f}_{\mathrm{y}}\right) \cdot \mathrm{f}_{\mathrm{c}}} \leq 0.25 \cdot \mathrm{f}_{\mathrm{c}} \mathrm{MPa} \\
\mathrm{k}: 0,6 \text { para hormigón colocado monolíticamente; } 0,5 \text { para hormigón colocado sobre hormigón ya endurecido, } \\
\text { con superficie rugosa. } \\
\text { k: } 0.6 \text { for monolithic cast concrete; } 0.5 \text { for concrete cast to hardened concrete with a rough surface. } \\
\lambda: \text { factor que tiene en cuenta el efecto del hormigón ligero. } \\
\lambda: \text { factor for considering the effect of lightweight concrete beams. }\end{array}$ \\
\hline
\end{tabular}

Notación:

$f_{c}$ : resistencia a compresión en probeta cilíndrica $/ f_{c}$ : compressive strength of the cylindrical specimen.

$f_{y}$ : límite elástico del acero de la armadura pasante / fy: reinforcement yield stress.

$\rho_{v}: A_{s} / A_{c}$, donde $A_{c}$ es el área de hormigón entre caras del plano de ensayo y $\mathrm{A}_{s}$ el área de la armadura pasante / $\rho_{v}: A_{s} / A_{c}$ where $A c$ is the area of concrete surface resisting shear friction and $A_{s}$ is the area of reinforcement crossing the surface.

$v_{\mathrm{n}}=$ tensión nominal de corte $/ v_{n}=$ nominal shear stress.

\section{PROGRAMA EXPERIMENTAL}

En este trabajo se diseñaron cuatro mezclas de hormigón: un hormigón convencional (HC-hormigón de control sin adición), un hormigón con un $50 \%$ de árido grueso reciclado (HR-hormigón reciclado), un hormigón convencional con la adición de un $8 \%$ de humo de sílice sobre el peso de cemento (HCS-hormigón de control con adición) y un hormigón con un $50 \%$ de árido grueso reciclado y la adición de un $8 \%$ de humo de sílice sobre el peso

\section{EXPERIMENTAL PROGRAM}

In this study, four concrete mixes were designed - a conventional concrete (CC-control concrete without additions), concrete with $50 \%$ recycled course aggregate (RC-recycled concrete), a conventional concrete with the addition of $8 \%$ silica fume referred to cement weight (CCS-control concrete with addition) and a concrete with $50 \%$ recycled aggregate and the addition of $8 \%$ of silica fume referred to cement weight (RCS-recycled concrete 
de cemento (HRS-hormigón reciclado con adición). Estos hormigones se caracterizaron a través de sus propiedades básicas y a continuación se abordaron ensayos de cortante-fricción en probetas sin armadura, con $2 \phi 5$ y con $2 \phi 6$ de armadura pasante (equivalentes a cuantías geométricas de $0,00 \%, 0,39 \%$ y $0,57 \%$, respectivamente), comparándose los resultados obtenidos con las formulaciones recogidas en el apartado anterior y con la norma española EHE (14).

\subsection{Materiales}

Los materiales empleados fueron los siguientes:

Cemento (C): CEM I - 42.5R (RC-05). Se analizaron las características físicas, químicas y mecánicas de las partidas de cemento utilizadas, resultando éstas adecuadas a la normativa.

Superfluidificante: para obtener consistencias blandas (cono de Abrams entre 5 y $10 \mathrm{~cm}$ ) se empleó un superfluidificante de alta eficacia a base de naftalenosulfonato condensado (S).

Áridos reciclados: se trabajó con material español procedente de demoliciones reales, de bajo control y clasificación única 0-40 mm (0-40R), formado fundamentalmente a partir de residuos de hormigón (72\%) y piedra (20\%). Las fracciones de áridos gruesos reciclados (RCA) se obtuvieron a partir de un proceso de cribado y lavado del material citado, obteniéndose así las fracciones 4-12 mm (4-12R) y 10-25 mm (10-25R), despreciándose las restantes (0-4R y $25-40 R)$.

Áridos naturales: como áridos naturales se utilizaron dos fracciones de árido grueso (NCA), catalogadas como cuarcitas filonianas, de $4-12 \mathrm{~mm}(4-12 \mathrm{C})$ y $10-25 \mathrm{~mm}$ (10-25C). El árido fino (FA) de 0-4 mm (0-4C) fue de origen calizo. Los áridos se sometieron a un proceso de lavado y cribado en planta. La Tabla 2 resume las propiedades físicas de los áridos.

Acero para armaduras: las barras de armadura utilizadas fueron del tipo B 500 SD (según EHE), con límite elástico característico de $500 \mathrm{MPa}$ (en ensayos $571 \mathrm{MPa}$ ) y límite de rotura en ensayos de $690 \mathrm{MPa}$.

Adición mineral: se utilizó Microsílice (MS) con tamaño de partículas comprendido entre 0,1 y 0,2 micras, un porcentaje de $\mathrm{SiO}_{2}$ del $94 \%$ y un Índice de Actividad medido (UNE 83-460-94) de 116,9\%. with addition). These concretes were first characterized in terms of their basic properties. Next, tests were performed to determine the shear friction in both nonreinforced specimens and specimens having a continuous reinforcement of $2 \phi 5$ or $2 \phi 6$ (equivalent, in geometric terms, to $0.00 \%, 0.39 \%$ and $0.57 \%$ respectively). The results of these tests were compared with the above formulas and with the Spanish standard EHE (14).

\subsection{Materials}

The materials used in this research were the following:

Cement (C): Portland CEM I - 42.5R cement (RC-05. Cement reception Directive. Ministry of Public Works 2008). An analysis was carried out on the physical, chemical and mechanical characteristics of the cement used. The results obtained were considered adequate taking the standards into account.

Water-reducing admixture: in order to obtain a workability defined by slump values between 5 and 10 $\mathrm{cm}$ a superplastizer $(S)$ was used.

Recycled aggregates: size fraction 0 to $40 \mathrm{~mm}(0-40 \mathrm{R})$ of Spanish material obtained from real demolition debris was used. It was made up principally of concrete (72\%) and stone (20\%). This material was washed and screened into two size fractions ( 4 to $12 \mathrm{~mm}, 4-12 R$, and 10 to $25 \mathrm{~mm}, 10-25 R$ ) that were used as recycled coarse aggregates $(R C A)$. The remaining size fractions were rejected (0-4R and 25-40R).

Natural aggregates: Two size fractions of crushed quartz (4 to $12 \mathrm{~mm}, 4-12 \mathrm{C}$ and 10 to $25 \mathrm{~mm}, 10-25 \mathrm{C}$ ) were used as natural coarse aggregates (NCA). Crushed limestone with a maximum size of $4 \mathrm{~mm}(0-4 C)$ was used as natural fine aggregate (FA). These natural aggregates were washed and screened in the plant. Table 2 summarizes the physical properties of the aggregates.

Reinforcement: reinforcing bars used were Spanish high ductility B $500 S D$ (according to EHE), with a yield point stress of $500 \mathrm{MPa}$ (tested $571 \mathrm{MPa}$ ) and a tensile strength of $690 \mathrm{MPa}$.

Mineral admixture (SF): Silica Fume containing particles ranging from 0.1 to 0.2 me in size, $94 \% \mathrm{SiO}_{2}$ and a $116.9 \%$ Activity Index (UNE 83-460-94) was used to make concretes with admixtures. 
Tabla 2 / Table 2

Propiedades físicas de los áridos utilizados.

Physical properties of the aggregates used.

\begin{tabular}{|c|c|c|c|c|c|c|c|}
\hline & EHE & $0-4 \mathrm{C}$ & $4-12 C$ & $10-25 \mathrm{C}$ & $4-12 R$ & $10-25 R$ & \\
\hline Shape index (UNE 7238:1971) & $(\geq 0.20)$ & - & 0.19 & 0.22 & 0.20 & 0.23 & Coeficiente de forma \\
\hline $\begin{array}{c}\text { Fineness modulus (UNE-EN 933- } \\
\text { 1:1998 and UNE-EN } \\
\text { 933-2/1M:1999) }\end{array}$ & - & 5.81 & 10.73 & 14.48 & 9.87 & 13.25 & Módulo granulométrico \\
\hline $\begin{array}{c}\text { Fine content (\% passing through } \\
\text { a } 0.063 \mathrm{~mm} \text { sieve) } \\
\text { (UNE-EN 933-1:1998) }\end{array}$ & $\begin{array}{l}\mathrm{AG}<1 \\
\mathrm{AF}<10\end{array}$ & $<8.8$ & $<0.1$ & $<0.1$ & $<0.2$ & $<0.2$ & $\begin{array}{c}\text { Finos (\% que pasa por el } \\
\text { tamiz } 0,063 \mathrm{~mm}\end{array}$ \\
\hline $\begin{array}{l}\text { Particle content less than } 4 \mathbf{~ m m} \\
\text { in size (\%) } \\
\text { (UNE-EN 933-2/1M:1999) }\end{array}$ & $<10$ & - & 1.5 & 0.4 & 4.7 & 0.6 & $\begin{array}{l}\text { Partículas de tamaño } \\
\text { inferior a } 4 \mathrm{~mm}(\%)\end{array}$ \\
\hline $\begin{array}{c}\text { Flakiness index (\%) (UNE-EN 933- } \\
\text { 1:1998 and UNE-EN } \\
\text { 933-2/1M:1999) }\end{array}$ & $(<35)$ & - & 25 & 11 & 9 & 7 & Índice de lajas (\%) \\
\hline $\begin{array}{c}\text { Density }\left(\mathbf{g} / \mathbf{c m}^{3}\right) \text { (UNE 83133: } \\
1990 \text { and UNE 83134: } \\
1990)\end{array}$ & - & 2.68 & 2.64 & 2.64 & 2.35 & 2.37 & Densidad $\left(\mathbf{g} / \mathrm{cm}^{3}\right)$ \\
\hline $\begin{array}{l}\text { Water saturated surface dry density } \\
\left(\mathbf{g} / \mathrm{cm}^{3}\right) \text { () (UNE 83133:1990 and } \\
\text { UNE 83134:1990) }\end{array}$ & - & 2.70 & 2.65 & 2.64 & 2.47 & 2.48 & $\begin{array}{l}\text { Densidad real saturada } \\
\text { superficie seca }\left(\mathrm{g} / \mathrm{cm}^{3}\right)\end{array}$ \\
\hline $\begin{array}{l}\text { Water absorption (\%) (UNE } \\
\text { 83133:1990 and UNE 83134: } \\
1990 \text { ) }\end{array}$ & $<5$ & 0.88 & 0.07 & 0.17 & 4.82 & 4.59 & Absorción (\%) \\
\hline $\begin{array}{c}\text { Porosity (\%) (UNE 83133:1990 } \\
\text { and UNE 83134: } \\
\text { 1990) }\end{array}$ & - & 2.36 & 0.19 & 0.45 & 11.33 & 10.88 & Porosidad (\%) \\
\hline $\begin{array}{c}\text { Abrasion resistance ("Los Angeles" } \\
\text { coefficient) (\%) } \\
\text { (UNE 1097-2:1999) }\end{array}$ & $(\leq 40)$ & - & 32 & 27 & 32 & 34 & $\begin{array}{c}\text { Dureza (Los Ángeles) } \\
(\%)\end{array}$ \\
\hline
\end{tabular}

\subsection{Dosificaciones}

Se establecieron como parámetros de diseño los correspondientes a un hormigón para armar sometido a una clase de exposición normal (IIa y IIb) y consistencia blanda (5-10 cm de asiento en Cono de Abrams), características habituales en el campo de la edificación española. Con este dato de partida, se establece, según la EHE (14): una relación W/C (agua total/material cementante) menor o igual que 0,55 (ratio que incluye el agua que aporta la humedad de los áridos, calculada previamente a la fabricación), un contenido de cemento mayor o igual que $300 \mathrm{~kg} / \mathrm{m}^{3}$, una resistencia a compresión a alcanzar en 28 días igual o superior a $30 \mathrm{MPa}$, y una consistencia blanda, definida por un asiento en cono de Abrams entre $5-10 \mathrm{~cm}$. Además, según los objetivos establecidos en el trabajo, se fija como cantidad de humo de sílice en las mezclas HCS y HRS un $8 \%$ en peso a añadir sobre el peso de cemento.

Tras numerosos tanteos se ajustaron los parámetros de dosificación (Tabla 3) según las siguientes pautas:

\subsection{Mix proportions}

Design parameters were established for reinforced concrete subjected to standard exposure levels (IIa and IIb) and consistency defined by slump values between 6 and $9 \mathrm{~cm}$, which are the standard ones used in the Spanish construction sector. Based on this information and in accordance with EHE (14) stipulations, the following data were obtained: W/C ratio (water) cementitious ratio) less than or equal to 0.55 . (this value includes the water from the aggregates due to their moisture content, which is calculated prior to production), cement content greater than or equal to $300 \mathrm{~kg} / \mathrm{m}^{3}$, compressive cylinder strength at 28 days of age greater than or equal to $30 \mathrm{MPa}$, and consistency defined by slump values between 6 and $9 \mathrm{~cm}$. In addition, and in accordance with the objectives of this project, the amount of silica fume in the CCS and RCS mixes to be added to the cement weight was fixed at $8 \%$.

Following numerous trials, the proportion parameters were determined (Table 3). 
- En el HC, ajuste final de la relación $\mathrm{W} / \mathrm{C}(\mathrm{W} / \mathrm{C}=0,55)$, para un contenido de cemento de $325 \mathrm{~kg} / \mathrm{m}^{3}$ y con una dosis de superfluidificante del $1,2 \%$ (superfluidificante/material-cementante en peso) teniendo en cuenta los objetivos de consistencia y resistencia apuntados.

- Ajuste del porcentaje de sustitución de áridos gruesos naturales por áridos gruesos reciclados al $50 \%$ (en volumen), en función de las resistencias a compresión mínimas a 28 días.

- Ajuste del contenido de cemento del HR para obtener consistencias similares al $\mathrm{HC}$, manteniendo el porcentaje de superfluidificante. Con este objetivo se aumentó la cantidad de cemento en un 6,2\% ( $C=345$ $\mathrm{kg} / \mathrm{m}^{3}$ ), resultado coincidente con los trabajos de otros autores (15).
- Final adjustment of the $W / C$ ratio $(W / C=0.55)$ with a cement content of $325 \mathrm{~kg} / \mathrm{m}^{3}$ and a superplasticizer percentage of $1.2 \%$ (referrer to binder weight) in the CC, taking into consideration consistency and strength objectives.

- Adjustment of the percentage of natural coarse aggregates replaced by recycled coarse aggregates to $50 \%$ (volume), based on the minimum compressive strength required at 28 days of age.

- Adjustment of the cement content in RC to obtain a consistency similar to CC while maintaining the percentage of superplasticizer. In order to do this, the amount of cement was increased by $6.2 \%$ ( $C=345$ $\left.\mathrm{kg} / \mathrm{m}^{3}\right)$, which agrees with the work of other authors (15).

Tabla 3 / Table 3

Parámetros básicos de dosificación. Basic proportion parameters.

\begin{tabular}{|c|c|c|c|c|c|c|c|c|c|c|c|c|}
\hline & $\begin{array}{c}0-4 \mathrm{C} \\
\% \\
\end{array}$ & $\begin{array}{c}4-12 C \\
\% \\
\end{array}$ & $\begin{array}{c}4-12 R \\
\% \\
\end{array}$ & $\begin{array}{c}10-25 C \\
\% \\
\end{array}$ & $\begin{array}{c}10-25 R \\
\% \\
\end{array}$ & $\begin{array}{c}\text { MS/C } \\
\% \\
\end{array}$ & $\begin{array}{c}\mathrm{C} \\
\mathrm{kg} / \mathrm{m}^{3} \\
\end{array}$ & $\begin{array}{l}W / C \\
\mathrm{~kg} / \mathrm{kg}\end{array}$ & $\begin{array}{c}\text { S/C } \\
\% \\
\end{array}$ & $\begin{array}{l}\mathrm{FA} / \mathrm{CA} \\
\mathrm{kg} / \mathrm{kg}\end{array}$ & $\begin{array}{c}\text { (NCA+RCA+ } \\
\text { NFA+MS)/C } \\
\mathrm{kg} / \mathrm{kg}\end{array}$ & \\
\hline CC & 100 & 100 & 0 & 100 & 0 & 0.0 & 325 & 0.55 & 1.35 & 0.97 & 6.08 & $\mathrm{HC}$ \\
\hline $\operatorname{ccs}$ & 100 & 100 & 0 & 100 & 0 & 8.0 & 325 & 0.55 & 1.19 & 0.87 & 5.44 & HCS \\
\hline RC & 100 & 50 & 50 & 50 & 50 & 0.0 & 345 & 0.55 & 1.26 & 0.96 & 5.43 & HR \\
\hline RCS & 100 & 50 & 50 & 50 & 50 & 8.0 & 345 & 0.55 & 1.24 & 0.86 & 4.84 & HRS \\
\hline
\end{tabular}

\subsection{Diseño de los ensayos e instrumentación}

La definición geométrica de la probeta (Figura 1) se basó en la empleada en la mayoría de estudios de cortantefricción recogidos en la bibliografía (16-24).

\subsection{Specimen design and instrumentation}

The geometric design of the specimen (Figure 1) was based on the one used most frequently in shear friction studies (16-24).

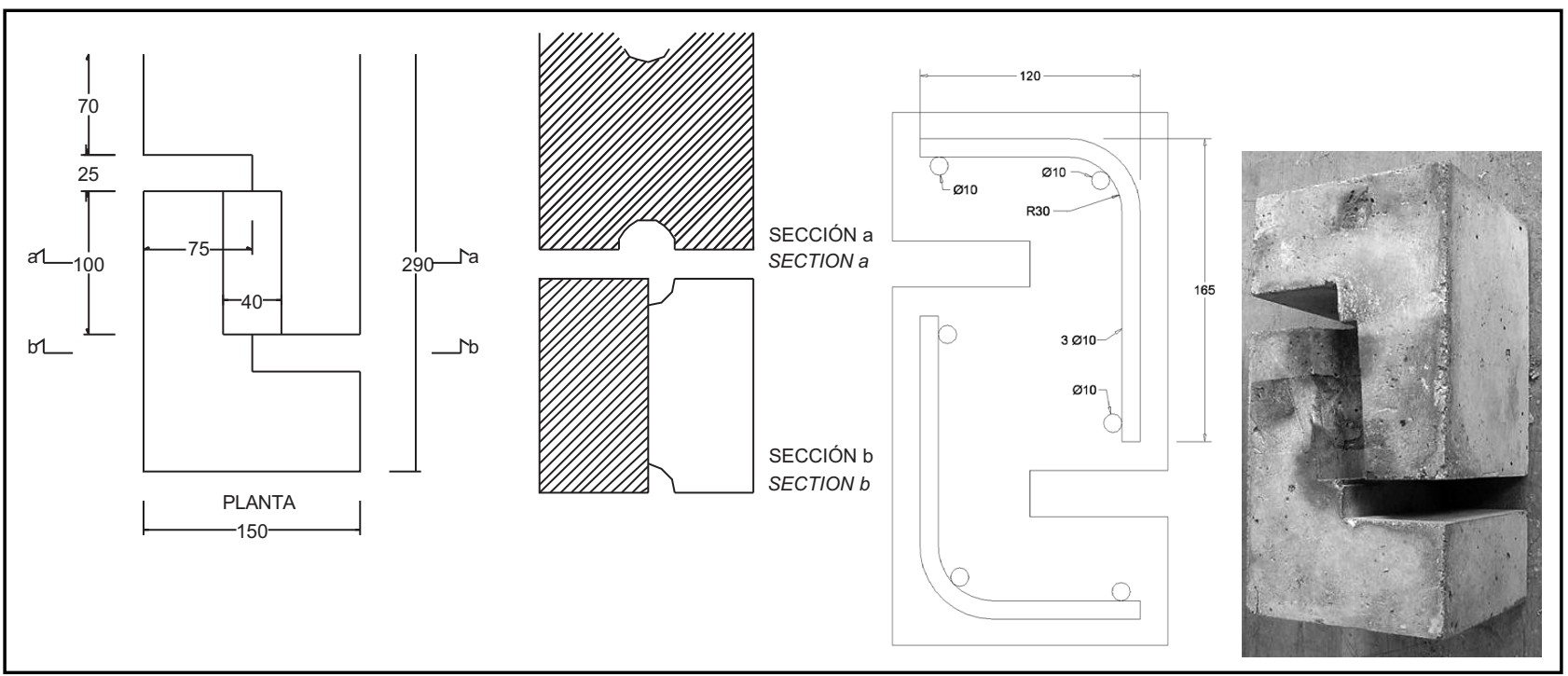

Figura 1. Dimensiones $(\mathrm{mm})$ y armado de las probetas doble-L.

Figure 1. Dimensions $(\mathrm{mm})$ and reinforcement of the double- $L$ shear specimen. 
Para la definición del armado (Figura 1) se analizaron diversos modelos biela-tirante, adaptando su disposición a las direcciones y magnitudes de las tensiones principales. De los modelos biela-tirante analizados se optó por el definido en la Figura 2.

Para cada uno de los hormigones estudiados y para las diferentes cuantías de armadura pasante $\left(\rho_{\mathrm{v}}=0,00 \%\right.$, $0,39 \%$ y $0,57 \%$ ) se realizaron ensayos hasta rotura bajo control de deformación. En el ensayo se programó una pre-compresión de $10 \mathrm{kN}$ tras la que iniciaba el control por deformación. A continuación, y hasta rotura, se aplicó un desplazamiento a velocidad de $1 \times 10^{-3} \mathrm{~mm} / \mathrm{s}$. Este desplazamiento se controló mediante transductores potenciométricos que se situaron mediante angulares adheridos a la superficie de la probeta y que permitieron la medida del desplazamiento relativo entre caras del plano de cortante durante todo el ensayo (Figura 3).

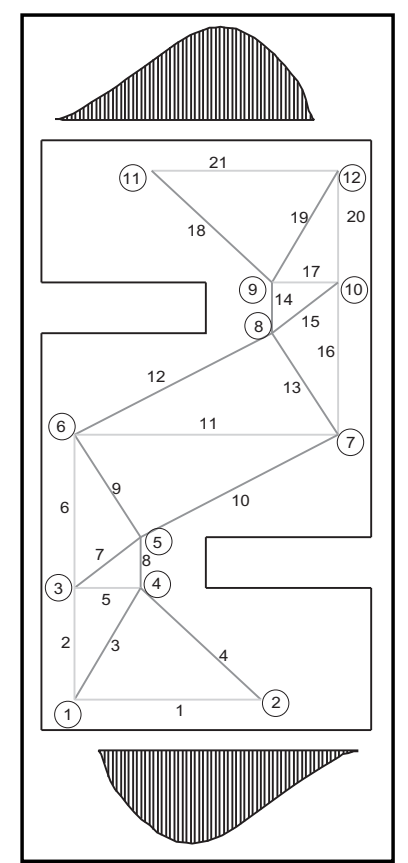

Figura 2. Modelo biela-tirante. Figure 2. Strut-ant-tie model.

\section{RESULTADO DE LOS ENSAYOS}

\subsection{Propiedades básicas}

Todas las mezclas presentaron consistencia blanda (cono de Abrams entre 6 y $9 \mathrm{~cm}$ ). Por otra parte, los resultados de resistencias a compresión, tracción indirecta y módulo de elasticidad estático a 28 días se muestran en la Tabla 4.
The geometric definition of the reinforced specimen (Figure 1) was based on an analysis of several different strut and tie models, STM, attempting to provide a faithful adaptation of the directions and magnitudes of the main stresses. After analyzing the different strut and tie models, we chose to use the one defined in Figure 2.

For each type of concrete studied and for the different quantities of continuous reinforcement $\left(\rho_{v}=0.00 \%\right.$, $0.39 \%$ y $0.57 \%)$, fracture tests were carried out in deformation control. The trial consisted of programming a pre-compression of $10 \mathrm{kN}$ after which the deformation control was set at zero. Next, and until fracture occurred, a displacement was applied at a rate of $1 \times 10^{-3} \mathrm{~mm} / \mathrm{s}$. This displacement was controlled by potentiometric transducers which were placed on an angular position attached to the specimen surface, which allowed the measurement of the relative displacement between the shear plane face throughout the trial (Figure 3).

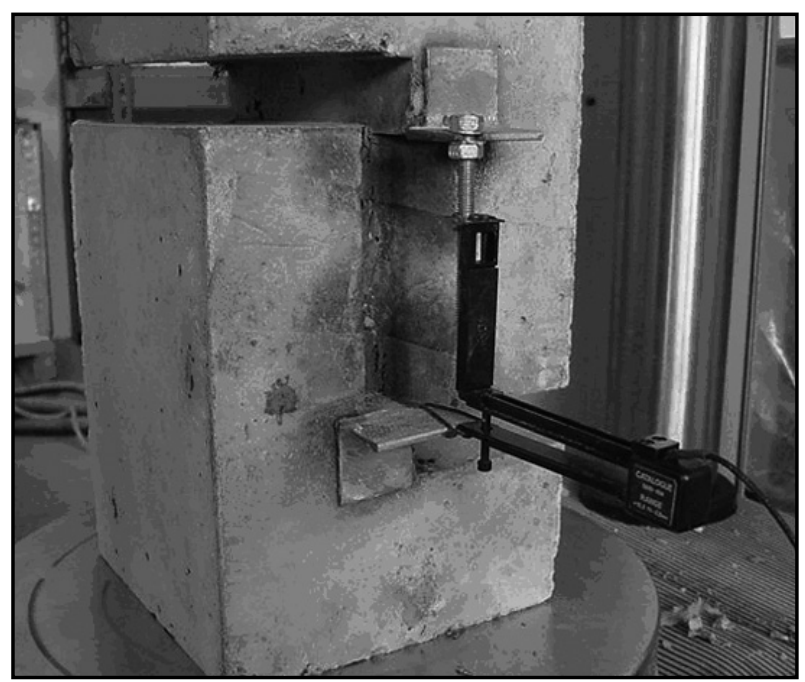

Figura 3. Instrumentación de la probeta doble - L.

Figure 3. Instrumentation of the double $-L$ shear specimen.

\section{TRIAL RESULTS}

\subsection{Basic properties}

All the mixes presented slump values ranging between 6 $\mathrm{cm}$ and $9 \mathrm{~cm}$. Otherwise, the results of compressive strength, tensile splitting strength and static modulus of elasticity at 28 days are shown in Table 4. 
Tabla 4 / Table 4

Propiedades mecánicas de los hormigones a 28 días.

Mechanical properties of the concretes at 28 days.

\begin{tabular}{|c|c|c|c|c|}
\hline & $\mathrm{f}_{\mathrm{c}, 28}(\mathrm{MPa})$ & $\mathrm{f}_{\mathrm{ct}, 28}(\mathrm{MPa})$ & $\mathrm{E}_{\mathrm{c}, 28}(\mathrm{MPa})$ & \\
\hline CC & 37.87 & 3.28 & 32992 & $\mathrm{HC}$ \\
\hline $\operatorname{ccs}$ & 38.13 & 3.26 & 32776 & HCS \\
\hline $\mathrm{RC}$ & 36.87 & 3.19 & 30045 & HR \\
\hline RCS & 38.75 & 2.98 & 30845 & HRS \\
\hline
\end{tabular}

Como puede observarse, el incremento en la cantidad de cemento de 325 a $345 \mathrm{~kg} / \mathrm{m}^{3}$ y el uso del $50 \%$ de árido grueso reciclado apenas sin humedad mantiene las resistencias a compresión de los hormigones reciclados (HR y HRS) comparables a las de los hormigones convencionales de control (HC y HCS, respectivamente (25)).

El efecto puzolánico de la adición de humo de sílice tiende a incrementar la resistencia a compresión de los hormigones que la incorporan. Así, los hormigones con el $8 \%$ de humo de sílice muestran mayores resistencias a compresión que sus correspondientes de control sin adición. Por otra parte, con esta adición mineral el HRS desarrolla una resistencia a compresión superior a la del HC.

Ni la inclusión de áridos reciclados ni la adición de humo de sílice suponen modificaciones en la resistencia a tracción de los hormigones estudiados. Estos resultados coinciden con los señalados por otros autores (26).

Finalmente, debido al mortero adherido al árido, los hormigones con menor módulo de elasticidad estático son aquellos que incorporan árido reciclado. Como en el caso de la resistencia a tracción indirecta, la adición de humo de sílice no modifica este parámetro. Estos resultados coinciden con los observados en la bibliografía (27-28).

\subsection{Cortante-fricción}

En la Tabla 5 se incluyen los valores medios del cortante-fricción obtenidos a partir de los ensayos para cada uno de los distintos hormigones empleados, HC, HCS, HR y HRS, y para las distintas cuantías de armado transversal utilizadas. En la Figura 4 se puede apreciar los modos de rotura tipo obtenidos con las probetas utilizadas.

En la Figura 5 se observa que para todas las cuantías de armado se detectan notables disminuciones del cortante de rotura en los hormigones reciclados. Estas disminuciones son sensiblemente mayores en el caso de probetas con ausencia de armadura pasante (20,7\% de disminución).
It can be seen that by increasing the amount of cement from 325 to $345 \mathrm{~kg} / \mathrm{m}^{3}$ and using $50 \%$ practically dry recycled aggregates the compressive strength observed in RC and RCS was found to be similar to that of CC and CCS respectively (25).

The pozzolanic effect of the silica fume tends to increase the compressive strength of the concretes with this mineral admixture. In this way, concretes containing $8 \%$ silica fume displayed greater compressive strength than control concretes that did not contain this admixture. Moreover, with this mineral admixture, RCS displayed greater compressive strengths than the CC.

Neither the inclusion of recycled aggregates nor the addition of silica fume had any particular impact on the tensile splitting strength of the concrete types analysed. These results coincide with those reported in the different studies (26).

Finally, due to the adhered mortar, specimens with the lowest static modulus of elasticity were those corresponding to concrete containing recycled aggregates. As with the tensile splitting strength, the addition of silica fume did not modify this parameter. Both these results coincide with those observed in the literature (27-28).

\subsection{Shear friction}

Table 5 presents the mean shear friction values resulting from the tests using the different types of concrete, CC, CCS, RC and RCS, and for the different quantities of transverse reinforcement used. In Figure 4 it is possible to see the fracture modes obtained with the specimens used.

In figure 5 it was seen that for all reinforcement quantities there was a substantial decrease in shear fracture in recycled concretes. These decreases were considerably higher in specimens without continuous reinforcement (a $20.7 \%$ decrease). In cases where continuous 
Tabla $5 / T$ able 5

Cortante-fricción último $(\mathrm{kN})$.

Ultimate shear friction $(\mathrm{kN})$.

\begin{tabular}{|c|c|c|c|c|}
\hline & $\rho_{v}=0.00 \%$ & $\rho_{v}=0.39 \%$ & $\rho_{v}=0.57 \%$ & \\
\hline $\mathrm{CC}$ & 62.18 & 88.00 & 93.26 & $\mathrm{HC}$ \\
\hline $\operatorname{ccs}$ & $\begin{array}{l}64.26 \\
(3.34)\end{array}$ & $\begin{array}{l}95.22 \\
(8.21)\end{array}$ & $\begin{array}{l}93.54 \\
(0.29)\end{array}$ & HCS \\
\hline RC & $\begin{array}{c}49.32 \\
(-20.68)\end{array}$ & $\begin{array}{c}78.68 \\
(-10.59)\end{array}$ & $\begin{array}{c}78.13 \\
(-16.23)\end{array}$ & HR \\
\hline RCS & $\begin{array}{l}65.92 \\
(6.00)\end{array}$ & $\begin{array}{c}84.71 \\
(-3.73)\end{array}$ & $\begin{array}{l}98.38 \\
(5.49)\end{array}$ & HRS \\
\hline
\end{tabular}

Nota: entre paréntesis (cortante-fricción - HC cortante-fricción) x 100 / HC cortante-fricción. / Note: in brackets (shear friction - CC shear friction) x 100 / CC Shear friction.

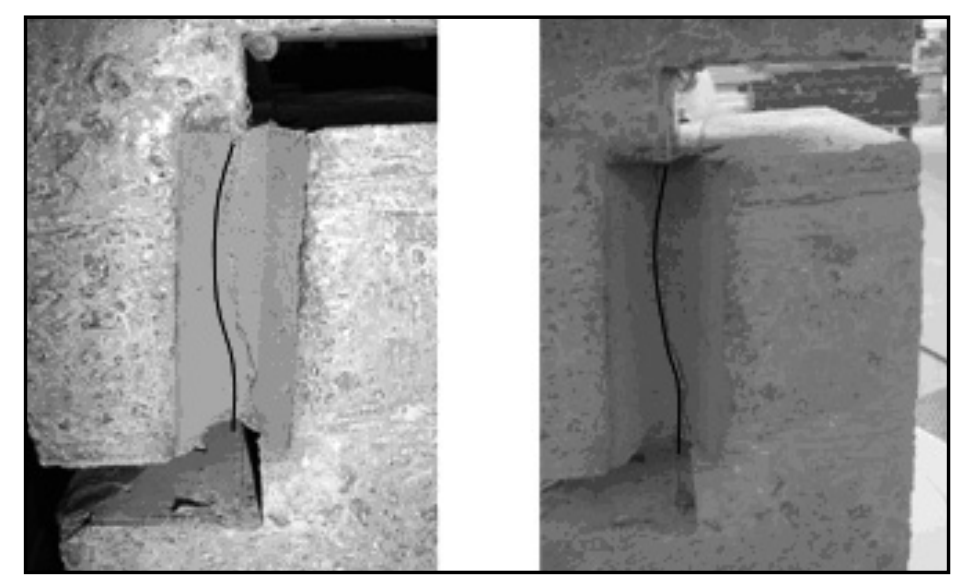

Figura 4. Modos de rotura.

Figure 4. Fracture modes.

En el caso de utilizar armadura pasante el valor de la disminución se reduce hasta un $10,6 \%$ y un $16,2 \%$ para cuantías de 0,39\% y 0,57\%, respectivamente. Esta disminución en la capacidad frente a cortante-fricción se debe a la presencia de mortero adherido al árido reciclado que hace que las fisuras intersecten a las partículas del árido además de a la matriz de cemento. Este efecto es mucho más importante en probetas sin armadura, lo que justifica los bajos valores de rotura obtenidos en este caso para el hormigón reciclado. reinforcement was used, the diminishing value was reduced by up to $10.6 \%$ and by $16.2 \%$ for quantities of $0.39 \%$ and $0.57 \%$ respectively. This decrease in shear friction capacity is due to the presence of mortar adhered to the recycled aggregate which causes the cracks to intersect the aggregate particles as well as the matrix. This effect takes on greater importance in the absence of reinforcement, which would explain the low fracture values obtained here for recycled concrete.

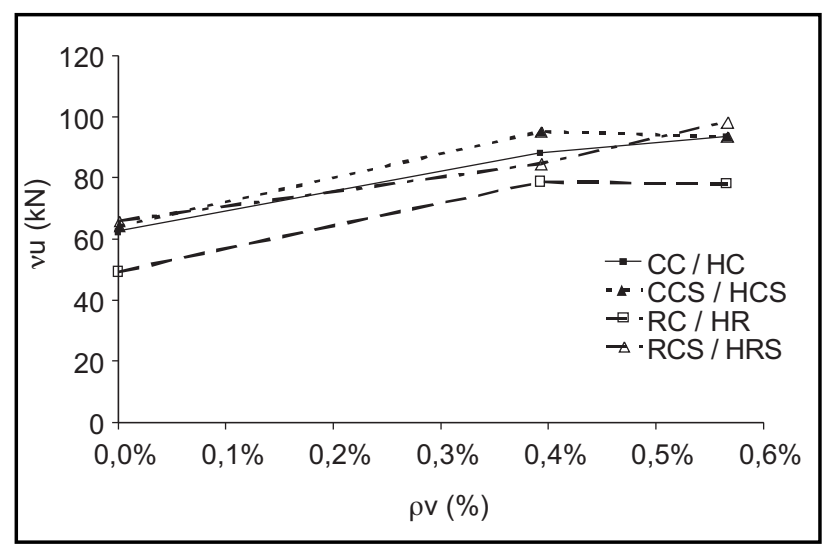

Figura 5. Cortante-fricción último $(\mathrm{kN})$.

Figure 5. Ultimate Shear friction (kN). 
La adición de humo de sílice contrarresta el efecto negativo del árido reciclado aumentando la capacidad de la matriz. De esta forma, los HCS presentan, excepto para la cuantía del 0,39\% (en donde se detecta una disminución de tan sólo el 3,7\%), mayor capacidad frente a cortante-fricción que los $\mathrm{HC}$.

Igualmente, se presentan las curvas cortante-deslizamiento del plano de rotura (Figuras 6, 7 y 8 ). En ellas se observa que las probetas sin armadura presentan curvas similares, aunque las de las probetas de hormigones reciclados con armadura pasante son sensiblemente diferentes al resto. La rama post-pico es siempre más tendida en estos hormigones, llegando a producirse largas "mesetas" que llevan a deformaciones en rotura considerablemente superiores a las de los hormigones convencionales y a las de los hormigones con humo de sílice (con o sin árido reciclado).

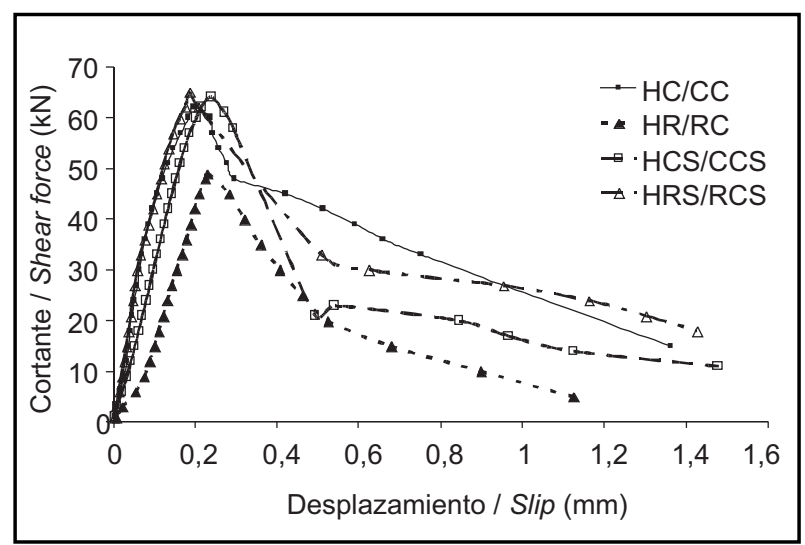

Figura 6. Solicitación de cortante-deslizamiento del plano de rotura. $\rho v=0.00 \%$.

Figure 6. Shear friction-Crack slip. $\rho v=0.00 \%$.
The addition of silica fume was found to offset the negative effect of the recycled aggregate, increasing the matrix capacity. Hence, with the exception of the quantity of $0.39 \%$ (where a decrease of only $3.7 \%$ was detected) the RCS showed a greater shear-friction capacity than the $C C$.

The shear load - shear slip curves are also presented (Figures 6, 7 and 8). In these curves it is possible to observe that the specimens without reinforcement behave in a like manner in terms of the shape of the shear-deformation curve. In the specimens with reinforcement, however, the curves pertaining to recycled concretes are substantially different from the rest. The post-peak curve is always more extended in these concretes and this ends up giving rise to long "plateaus" which cause fracture deformations (for the ultimate load) that are considerably higher than those of conventional concretes and concretes with silica fume (with or without recycled aggregate).

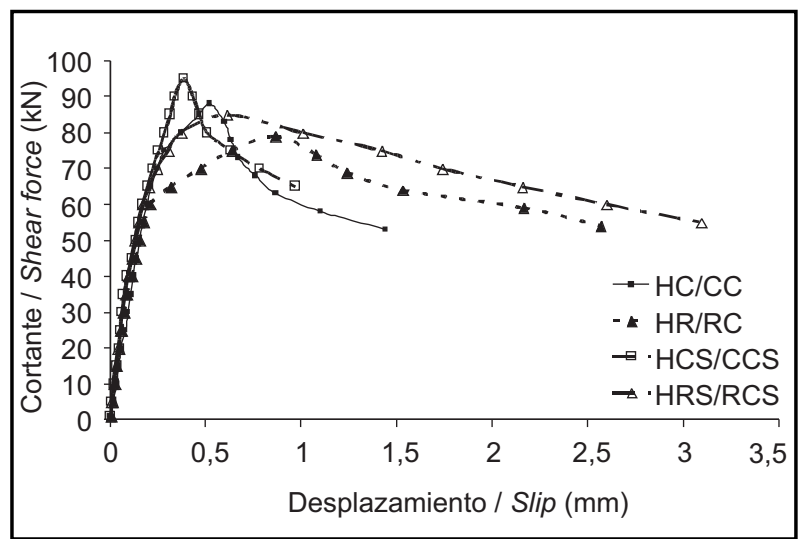

Figura 7. Solicitación de cortante-deslizamiento del plano de rotura. $\rho v=0.39 \%$.

Figure 7. Shear friction-Crack slip. $\rho v=0.39 \%$.

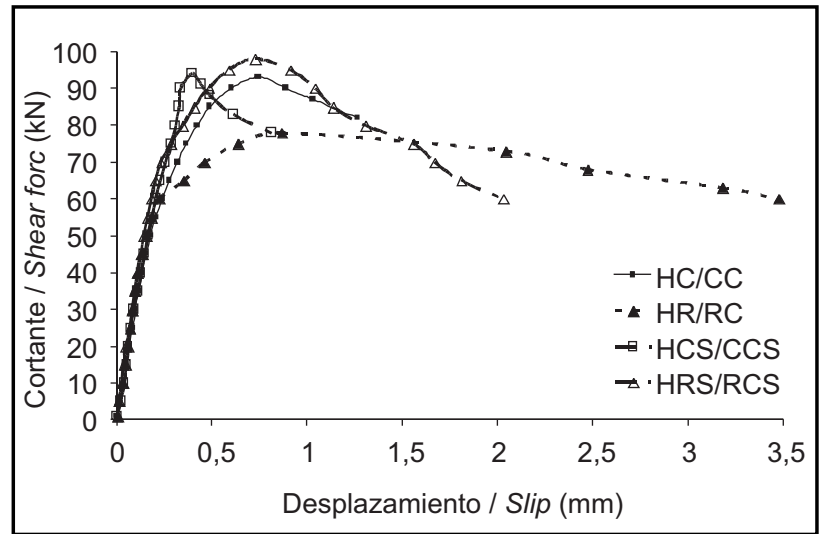

Figura 8. Solicitación de cortante-deslizamiento del plano de rotura. $\rho v=0.57 \%$.

Figure 8. Shear friction-Crack slip. $\rho v=0.57 \%$. 


\section{COMPARACIÓN ENTRE LAS DIVERSAS FORMULACIONES}

Para el hormigón reciclado no existen, en la actualidad, estudios que ofrezcan modelos de ajuste incorporables en las normativas vigentes. No existen, por tanto, expresiones específicas para estos hormigones que describan su respuesta. Consecuentemente se han utilizado las formulaciones descritas en el apartado 3 para valorar su bondad a la vista de los resultados experimentales. Los resultados que estas formulaciones proporcionan, así como la ratio cortante experimental versus cortante último teórico (margen de seguridad), se recogen en la Tabla 6.

\section{COMPARISONS USING THE DIFFERENT FORMULAS}

Today, for the recycled concretes, there are not studies that offer models of adjustment to be incorporated in the codes. Therefore, specific expressions that describe the performance of these concretes do not exist. So, the expressions described in section 3 of this paper were used to assess their fitness to the experimental results. The results provided by these expressions, in addition to the experimental shear ratio versus the theoretical shear friction are given in table 6.

Tabla 6 / Table 6

Cortante último experimental versus cortante último teórico $(\mathrm{kN})$. Experimental shear friction versus predicted shear friction $(k N)$.

\begin{tabular}{|c|c|c|c|c|c|c|c|c|c|c|c|c|}
\hline & $\rho_{v}(\%)$ & $\begin{array}{c}\mathbf{V}_{\exp }(\mathrm{kN}) \\
\text { Birkeland } \\
{[1]}\end{array}$ & $\begin{array}{c}\text { Raths } \\
\text { [2] }\end{array}$ & $\begin{array}{c}\text { Shaikh } \\
{[3]}\end{array}$ & $\begin{array}{c}\text { Mattok } \\
{[4]}\end{array}$ & $\begin{array}{c}\text { Walraven } \\
{[5]}\end{array}$ & $\begin{array}{c}\text { Mattok } \\
\text { [6] }\end{array}$ & $\begin{array}{c}\text { Mau y } \\
\text { Hsu's [7] }\end{array}$ & $\begin{array}{c}\text { Loov y } \\
\text { Patnaik } \\
\text { [8] }\end{array}$ & EHE (14) & & \\
\hline \multirow{3}{*}{ Cc } & 0.00 & 62.19 & & & & $\begin{array}{l}28.00 \\
(2.22)\end{array}$ & & $\begin{array}{l}33.84 \\
(1.84)\end{array}$ & & & $\begin{array}{l}22.19 \\
(2.80)\end{array}$ & \multirow{3}{*}{$\mathrm{HC}$} \\
\hline & 0.39 & 88.00 & $\begin{array}{l}33.38 \\
(2.64)\end{array}$ & $\begin{array}{l}43.58 \\
(2.02)\end{array}$ & $\begin{array}{l}43.55 \\
(2.02)\end{array}$ & $\begin{array}{l}43.71 \\
(2.01)\end{array}$ & $\begin{array}{l}47.23 \\
(1.85)\end{array}$ & $\begin{array}{l}49.55 \\
(1.78)\end{array}$ & $\begin{array}{l}56.91 \\
(1.55)\end{array}$ & $\begin{array}{l}53.04 \\
(1.66)\end{array}$ & $\begin{array}{l}35.34 \\
(2.49)\end{array}$ & \\
\hline & 0.57 & 93.26 & $\begin{array}{l}48.07 \\
(1.94)\end{array}$ & $\begin{array}{l}52.30 \\
(1.78)\end{array}$ & $\begin{array}{l}52.26 \\
(1.78)\end{array}$ & $\begin{array}{l}50.62 \\
(1.84)\end{array}$ & $\begin{array}{l}56.72 \\
(1.64)\end{array}$ & $\begin{array}{l}56.46 \\
(1.65)\end{array}$ & $\begin{array}{l}68.30 \\
(1.37)\end{array}$ & $\begin{array}{l}63.18 \\
(1.48)\end{array}$ & $\begin{array}{l}50.90 \\
(1.83)\end{array}$ & \\
\hline \multirow{3}{*}{$\operatorname{ccs}$} & 0.00 & 62.24 & & & & $\begin{array}{l}28.00 \\
(2.22)\end{array}$ & & $\begin{array}{l}33.97 \\
(1.83)\end{array}$ & & & $\begin{array}{l}21.97 \\
(2.83)\end{array}$ & \multirow{3}{*}{ HCS } \\
\hline & 0.39 & 95.22 & $\begin{array}{l}33.38 \\
(2.85)\end{array}$ & $\begin{array}{l}43.58 \\
(2.19)\end{array}$ & $\begin{array}{l}43.55 \\
(2.19)\end{array}$ & $\begin{array}{l}43.71 \\
(2.18)\end{array}$ & $\begin{array}{l}47.39 \\
(2.01)\end{array}$ & $\begin{array}{l}49.68 \\
(1.92)\end{array}$ & $\begin{array}{l}57.11 \\
(1.67)\end{array}$ & $\begin{array}{l}53.22 \\
(1.79)\end{array}$ & $\begin{array}{l}35.34 \\
(2.69)\end{array}$ & \\
\hline & 0.57 & 93.54 & $\begin{array}{l}48.07 \\
(1.95)\end{array}$ & $\begin{array}{l}52.30 \\
(1.79)\end{array}$ & $\begin{array}{l}52.26 \\
(1.79)\end{array}$ & $\begin{array}{l}50.62 \\
(1.85)\end{array}$ & $\begin{array}{l}56.94 \\
(1.64)\end{array}$ & $\begin{array}{l}56.59 \\
(1.65)\end{array}$ & $\begin{array}{l}68.53 \\
(1.36)\end{array}$ & $\begin{array}{l}63.39 \\
(1.48)\end{array}$ & $\begin{array}{l}50.90 \\
(1.84)\end{array}$ & \\
\hline \multirow{3}{*}{ RC } & 0.00 & 49.33 & & & & $\begin{array}{l}28.00 \\
(1.76)\end{array}$ & & $\begin{array}{l}33.35 \\
(1.48)\end{array}$ & & & $\begin{array}{l}21.88 \\
(2.25)\end{array}$ & \multirow{3}{*}{ HR } \\
\hline & 0.39 & 78.68 & $\begin{array}{l}33.38 \\
(2.36)\end{array}$ & $\begin{array}{l}43.58 \\
(1.81)\end{array}$ & $\begin{array}{l}43.55 \\
(1.81)\end{array}$ & $\begin{array}{l}43.71 \\
(1.80)\end{array}$ & $\begin{array}{l}46.59 \\
(1.69)\end{array}$ & $\begin{array}{l}49.06 \\
(1.60)\end{array}$ & $\begin{array}{l}56.16 \\
(1.40)\end{array}$ & $\begin{array}{l}52.33 \\
(1.50)\end{array}$ & $\begin{array}{l}35.34 \\
(2.23)\end{array}$ & \\
\hline & 0.57 & 78.13 & $\begin{array}{l}48.07 \\
(1.63)\end{array}$ & $\begin{array}{l}52.30 \\
(1.49)\end{array}$ & $\begin{array}{l}52.26 \\
(1.49)\end{array}$ & $\begin{array}{l}50.62 \\
(1.54)\end{array}$ & $\begin{array}{l}55.87 \\
(1.40)\end{array}$ & $\begin{array}{l}55.97 \\
(1.40)\end{array}$ & $\begin{array}{l}67.39 \\
(1.16)\end{array}$ & $\begin{array}{l}62.34 \\
(1.25)\end{array}$ & $\begin{array}{l}50.90 \\
(1.54)\end{array}$ & \\
\hline \multirow{3}{*}{ RCS } & 0.00 & 65.92 & & & & $\begin{array}{l}28.00 \\
(2.35)\end{array}$ & & $\begin{array}{l}34.27 \\
(1.92)\end{array}$ & & & $\begin{array}{l}19.91 \\
(3.31)\end{array}$ & \multirow{3}{*}{ HRS } \\
\hline & 0.39 & 84.71 & $\begin{array}{l}33.38 \\
(2.54)\end{array}$ & $\begin{array}{l}43.58 \\
(1.94)\end{array}$ & $\begin{array}{l}43.55 \\
(1.95)\end{array}$ & $\begin{array}{l}43.71 \\
(1.94)\end{array}$ & $\begin{array}{l}47.78 \\
(1.77)\end{array}$ & $\begin{array}{l}49.98 \\
(1.69)\end{array}$ & $\begin{array}{l}57.57 \\
(1.47)\end{array}$ & $\begin{array}{l}53.65 \\
(1.58)\end{array}$ & $\begin{array}{l}35.34 \\
(2.40)\end{array}$ & \\
\hline & 0.57 & 98.38 & $\begin{array}{l}48.07 \\
(2.05)\end{array}$ & $\begin{array}{l}52.30 \\
(1.88)\end{array}$ & $\begin{array}{l}52.26 \\
(1.88)\end{array}$ & $\begin{array}{l}50.62 \\
(1.94)\end{array}$ & $\begin{array}{c}57.46 \\
(1.71)\end{array}$ & $\begin{array}{l}56.89 \\
(1.73)\end{array}$ & $\begin{array}{l}69.08 \\
(1.42)\end{array}$ & $\begin{array}{l}63.91 \\
(1.54)\end{array}$ & $\begin{array}{l}50.90 \\
(1.93)\end{array}$ & \\
\hline
\end{tabular}

Nota: entre paréntesis (cortante experimental / cortante teórico) / Note: In brackets (experimental shear / code shear).

Los valores que ofrecen las distintas formulaciones, para todos los hormigones considerados, resultan conservadores. Sin embargo, los hormigones reciclados (HR) presentan márgenes de seguridad más ajustados que los de los convencionales $(\mathrm{HC})$, diferencia que corrige la adición de humo de sílice al comparar los hormigones HCS y HRS.
The results obtained using the different formulations and for all the types of concretes under consideration were conservative. However, there is a noticeable difference between the safety margins offered by concretes made with recycled aggregate and those obtained with conventional concretes. As regards recycled concretes, the margins have a better fit than those of conventional ones. The addition of silica fume increases the ratios in recycled concretes with silica fume. 
Estos resultados indican que la presencia de mortero adherido al árido implica un peor comportamiento de los hormigones reciclados con el $50 \%$ de sustitución de la fracción gruesa frente al fenómeno de cortante-fricción, apareciendo, en numerosas ocasiones, roturas que afectan al árido reciclado. En este sentido, para mantener los márgenes de seguridad de los hormigones convencionales podría ser necesario establecer coeficientes correctores en los hormigones reciclados, al igual que se hace con los hormigones que contienen áridos ligeros o los hormigones de alta resistencia, en los que los mecanismos de rotura son sensiblemente diferentes a los que se producen en los hormigones convencionales. A la vista de los resultados, y para un $50 \%$ de sustitución de la fracción gruesa, el coeficiente corrector que es necesario aplicar para alcanzar los niveles de seguridad que exhiben los hormigones convencionales es de 0,85.

Los numerosos estudios llevados sobre hormigones con árido reciclado, sustituyendo un $20 \%$ de la fracción gruesa, han determinado su práctica equivalencia con los hormigones convencionales sin árido reciclado de resistencias de hasta $40 \mathrm{MPa}$. Esta equivalencia se constata tanto en las propiedades materiales (resistencias a tracción y compresión, cansancio a compresión y tracción, fluencia, retracción, etc.) como estructurales (flexión, cortante, adherencia, etc.) (29). Este hecho permite extrapolar de modo coherente la misma situación para el esfuerzo de cortante fricción, lo que ofrece una pauta de predicción de la corrección a efectuar en el intervalo 20$50 \%$. La interpolación lineal, en esta situación, resulta conservadora teniendo presente que numerosos autores extienden la equivalencia hasta la sustitución del $30 \%$ de árido grueso reciclado.

\section{CONCLUSIONES}

- En ausencia de armadura pasante, el cortante fricción último sufre una disminución del $20 \%$ cuando se emplea hormigón reciclado con una sustitución del $50 \%$ del árido grueso por árido reciclado. Esta pérdida se reduce al $10-15 \%$ al introducirse armadura pasante en diferentes cuantías.

- El empleo de humo de sílice supone un aumento considerable de la carga última de los hormigones reciclados, equiparándose las cargas de rotura frente a cortante fricción con las de los hormigones de control con árido natural y humo de sílice. Este efecto del humo de sílice no se detecta en los hormigones convencionales, por lo que cabe deducir que esta adición mejora la interfase matriz-árido reciclado.

- En cuanto a las deformaciones, los diferentes materiales presentan un comportamiento similar durante
In this way, to maintain the safety margins of conventional concretes, a correction factor might be necessary when recycled concretes are used, as is done with concrete containing lightweight aggregate and with high strength concrete, where the fracture mechanisms are significantly different from those produced in conventional concrete. As it can be seen, when $50 \%$ of replacement of the coarse aggregate is used, the correction factor that should be applied to achieve the security levels that exhibit the conventional concretes is 0.85 .

Many studies carried on with recycled concretes with $20 \%$ of recycled coarse aggregate and up to $40 \mathrm{MPa}$ of compressive strength, have shown that their behaviour is similar to that of conventional concretes. This equivalence is evident in both the basic properties (tensile splitting strength and compressive strength, behaviour under sustained compression and tensile load, creep, shrinkage, etc.) as in the structural behavior (bending, shear, adhesion, etc.) (29). This fact allows extrapolating consistently the same situation for the shear-friction behavior, providing a guideline for predicting the correction to be made in the range 20$50 \%$. In this situation, linear interpolation is conservative, taking into account that many authors consider the equivalence up to $30 \%$ of replacement with recycled coarse aggregate.

\section{CONCLUSIONS}

- Without reinforcement crossing the surface shear friction capacity of recycled concrete with $50 \%$ of recycled coarse aggregate suffers a decrease of $20 \%$. This loss comes down to $10-15 \%$ when any quantity of reinforcement is used.

- The use of silica fume led to a substantial rise in the ultimate load of recycled concretes, so that regardless of whether or not continuous reinforcement was used, the ultimate fracture loads of recycled concretes with silica fume were similar to the loads obtained with conventional concretes and with conventional concretes with silica fume. The effect of the silica fume is not detected in the conventional concretes, so it is necessary to deduce that this addition improves the interface cement paste-recycled aggregate.

- In terms of strains, all the different materials behaved similarly during the load process, with differences 
el proceso de carga y sólo en rotura aparecen diferencias, detectándose deformaciones sensiblemente mayores en los hormigones reciclados empleados.

- Los hormigones reciclados con humo de sílice presentan deformaciones en rotura considerablemente inferiores a las de los hormigones reciclados. El motivo, nuevamente, apunta a la ya aludida mejora de la interfase.

- Las formulaciones utilizadas para contrastar los resultados obtenidos experimentalmente evidencian, en todos los casos, márgenes de seguridad superiores a la unidad. Cabe destacar, sin embargo, el hecho de que las cargas de rotura que predicen algunas de las formulaciones se aproximan sensiblemente a las obtenidas experimentalmente cuando se emplea hormigón reciclado con el $50 \%$ de sustitución de la fracción gruesa. En estos casos, afectando al valor predicho por la expresión del coeficiente corrector 0,85 , se consigue alcanzar los mismos márgenes de seguridad que presentan los hormigones convencionales. Aceptando de modo conservador que el comportamiento de los hormigones no se ve modificado para sustituciones de hasta el $20 \%$ del árido grueso (lo que implica el valor unidad del coeficiente corrector) puede estimarse lineal la variación de tal coeficiente entre los porcentajes del $20 \%$ y $50 \%$ de sustitución. Para porcentajes superiores es necesario desarrollar una campaña experimental más amplia.

\section{AGRADECIMIENTOS}

Este estudio es parte del proyecto titulados "3.2358/2005/3-B: Estudio experimental preformativo sobre la utilización de los RCDs en hormigón reciclado de aplicación estructural (RECNHOR)", financiado por el Ministerio de Medio Ambiente español. La continuidad de esta línea de investigación se garantiza con el Nuevo proyecto CLEAM, Construcción Limpia, Eficiente y Amigable con el Medio ambiente, proyecto CENIT en colaboración con empresas y centros de investigación. appearing only in situations of fracture, where strains were slightly higher in recycled concretes.

- The recycled concretes with silica fume shown considerable lower ultimate strains values than recycled concretes. That is due to the improvement of the interface cement paste-recycled aggregate.

- The equations used to confirm the results obtained experimentally indicate, in all the cases, safety margins greater than the unit. Nevertheless, it is necessary to stand out the fact that, when recycled concrete with $50 \%$ of recycled coarse aggregate is used, the ultimate loads obtained with some of the equations come closer to those obtained experimentally. In these cases, affecting the predicted value with a corrector coefficient of 0.85 the same safety margins of those achieved with conventional concretes are reached. Accepting, conservatively, that the behavior of the recycled concretes is not modified for substitutions up to $20 \%$ of the coarse aggregate (what implies a corrector coefficient of the unit) the variation of such a coefficient between the percentages of $20 \%$ to $50 \%$ of substitution can be considered linear. For other percentages it is necessary to develop a wider experimental study.

\section{ACKNOWLEDGMENTS}

This study is part of the project entitled "3.2358/2005/3-B: An experimental pre-normative study on the use of $R C D s$ in recycled concrete for structural applications (RECNHOR)", financed by the Spanish Environmental Ministry. The future of this investigation is guarantied with the new project CLEAM, Construction clean, efficient and friendly with the environment; project CENIT in collaboration with companies and investigation centres.

\section{BIBLIOGRAFÍA / BIBLIOGRAPHY}

(1) Walraven, J. C.: "Fundamental Analysis of Aggregate Interlock", ASCE, Journal of the Structural Division, vol. 107, ST11 (noviembre 1981), pp. 2245-2270.

(2) Peng, L.: Shear Strength of Beams by Shear-Friction, Tesis de la University of Calgary, Alberta, enero 1999, p. 164.

(3) Tozser, O.: Shear Analysis Using Shear Friction-size Effect and Prestressing, Tesis de la University of Calgary, Alberta, septiembre 1998, p. 193.

(4) Walraven J. C.: "High Performance Concrete: Exploring a new material", Structural Engineering International, vol. 5, no 3 (agosto 1995), pp. 182-187.

(5) Walraven, J. C.: "Shear Friction in high Strength Concrete", Progress in Concrete Research, Delft University of Technology, vol. 4 (marzo 1995), pp. 57-65.

(6) Birkeland, P. W., Birkeland, H. W.: "Connections in Precast concrete construction", ACI Journal, vol. 63, no 3 (marzo 1966), pp. 345368. 
(7) Raths, C. H.: Reader Comments on "Designs Proposals for Reinforced Concrete Corbels" by A.H. Mattock; PCI Journal, vol. 22 , n 2 (marzo-abril 1977), pp. 93-98.

(8) Shainkh, A. F.: "Proposed Revisions to Shear-Friction Provisions", PCI Journal, vol. 23, no 2 (marzo-abril 1978), pp. $12-21$.

(9) Mattock, A. H.: "Shear Transfer in Concrete having reinforcement at an angle to the shear Plane", ACI Special Publication, vol. 42 (enero 1974), pp. 17-42.

(10) Walraven, J. C.; Frenay, J.; Pruijssers, A.: "Influence of concrete strength and Load History on the Shear Friction Capacity of Concrete Members", PCI Journal, vol. 32, no 1 (1987), pp. 66-84.

(11) Mattock, A. H.: Reader Comments on "Influence of concrete strength and Load History on the Shear Friction Capacity of Concrete Members", by J. C. Walraven, J. Frenay \& A. Pruijssers, PCI Journal, vol. 33, n 1 (enero-febrero 1988), pp. 165-166.

(12) Mau, S. T., Hsu, T. T. C.: Reader comments on "Influence of concrete strength and Load History on the Shear Friction Capacity of Concrete Members", by J. C. Walraven, J. Frenay \& A. Pruijssers, PCI Journal, vol. 33, no 1 (enero-febrero 1988), pp. 166-168.

(13) Loov, R. E., Patnaik, A. K.: "Horizontal Shear Strength of Composite Concrete Beams with a Rough Interface", PCI Journal, vol. 39, no 1 (enero-febrero 1994), pp. 48-69.

(14) EHE. Instrucción de Hormigón Estructural. Publicaciones del Ministerio de Fomento. Secretaría General Técnica.

(15) Ravindrarajah, R. S.; Tam, C. T.: "Methods of Improving the Quality of Recycled Aggregate Concrete"; Demolition and Reuse of Concrete and Masonry, vol. 2 Reuse of Demolition Waste, Proceedings of the Second International RILEM Symposium, Ed. Y. Kasai; (noviembre 1988), pp 575-584, ISBN 0-412-32110-6.

(16) Banta, T. E.: Horizontal Shear Transfer Between Ultra High Performance Concrete and Lightweight concrete, p. 130, thesis submitted to the faculty of the Virginia Polytechnic Institute, febrero 2005.

(17) Hwang, S. J.; Yu, H. W.; Lee, H. J.: "Theory of interface shear capacity of reinforced concrete", Journal of Structural Engineering, vol. 126, no 6 (junio 2000), pp. 700-707. doi:10.1061/(ASCE) 0733-9445 (2000)126:6 (700)

(18) Ibell, T.; Burgoyne, C.: "Use of fiber-reinforced plastics versus steel for shear reinforcement of concrete", ACI Structural Journal, vol. 96, no 6 (noviembre-diciembre 1999), pp. 997-1002.

(19) Balaguru, P.; Dipsia, M. G.: "Properties of fiber reinforced high-strength semilightweight concrete", ACI Materials Journal, vol. 90, no 5 (septiembre-octubre 1993), pp. 399-405.

(20) Duthinh, D.; Carino, N. J.: Shear design of high-strength concrete beams: a review of the state-of-the art, p. 198, en Report NISTIR 5870, Building and Fire Research Laboratory, National Institute of Standards and Technology, agosto 1996.

(21) Duthinh, D.: "Sensitivity of shear strength of reinforced concrete and prestressed concrete beams to shear friction and concrete softening according to modified compression field theory", ACI Structural Journal, vol. 96, no 4 (julio-agosto 1999), pp. 495-508.

(22) Mohamed, A.; Richard, N.: "Enhanced contact model for shear friction of normal and high-strength concrete", ACI Structural Journal, vol. 96, no 3 (mayo-junio 1999), pp. 348-360.

(23) Ince, R.; Yalcin, E.; Arslan, A.: "Size-dependent response of dowel action in R.C. members"; Engineering Structures, vol. 29 (2007), pp. 955-961. doi:10.1016/j.engstruct.2006.07.006

(24) Menkulasi, F.: Horizontal Shear Connectors for Precast Prestressed Bridge Deck Panels, p. 138, thesis submitted to the faculty of the Virginia Polytechnic Institute; agosto 2002.

(25) Rahal, K.: "Mechanical properties of concrete with recycled coarse aggregate", Building and Environmental, vol. 42, no 1 (enero 2005), pp. 407-415.

(26) Hendriks, Ch. F.; Pietersen, H. S.; Fraay, A. F. A.: "Recycling of Buildings and Demolition Waste - An Integrated approach". Use of Recycled Concrete Aggregate, Sustainable Construction; Ed. Dhir, Henderson y Limbachiya; pp. 419-432, 1998, ISBN 0-7277-2726-5.

(27) Carrasquillo, R. L.; Slate, F. O.; Nilson, A. H.: "Properties of High Strength Concrete Subject to Short - term Loading", ACI journal, vol. 78, no 3 (mayo-junio 1981), pp. 171-178.

(28) Hansen, T. C.; Narud, H.: "Strength of Recycled Concreted Made from Crushed Concrete Coarse Aggregate"; Concrete International, vol. 5, no 1 (enero 1983), pp. 79-83.

(29) Comisión 2. Grupo de trabajo 2/5 "Hormigón Reciclado": Utilización de árido reciclado para la fabricación de hormigón estructural, p. 189, Ed. ACHE (Asociación Científico-técnica del Hormigón Estructural), España, 2006. ISBN 84-89670-55-2. 